\title{
Dynamical Localization: Hydrogen Atoms in Magnetic and Microwave fields
}

\author{
Francesco Benvenuto, Giulio Casati(a) \\ Dipartimento di Fisica dell'Università, Via Castelnuovo 7, 22100 Como, Italy \\ Dima L. Shepelyansky $(b)$ \\ Laboratoire de Physique Quantique, UMR C5626 du CNRS, \\ Université Paul Sabatier, 31062, Toulouse, France
}

(11 September 1996)

\begin{abstract}
We show that dynamical localization for excited hydrogen atoms in magnetic and microwave fields takes place at quite low microwave frequency $\left(\omega n^{3} \ll 1\right)$. The estimates of localization length are given for different parameter regimes, showing that the quantum delocalization border drops significantly as compared to the case of zero magnetic field. This opens up broad possibilities for laboratory investigations.
\end{abstract}

P.A.C.S.: $36.10 .-\mathrm{k}, 05.45 .+\mathrm{b}$

Typeset using REVTEX 
Quantum dynamical localization is one of the most interesting phenomena within the so-called field of "Quantum Chaos" 四 . This phenomenon, which consists in the quantum suppression of classically chaotic diffusive process, was discovered long ago [2], and has also been observed in several laboratory experiments [3 -7]. Even though experimental results are in satisfactory agreement with theoretical predictions, the range of parameters over which quantum localization has been observed is still quite narrow. For example, in experiments performed on hydrogen and Rydberg atoms in microwave fields [3 5], in order to observe localization of the quantum probability distribution around the initially excited state $n_{0}$, it is necessary that the localization length $\ell_{\phi}$ of the distribution, measured in number of photons, be smaller than the number $N_{I}$ of photons required for ionization. Such a condition imposes severe experimental restrictions. Indeed, $N_{I}=\left(2 n_{0}^{2} \omega\right)^{-1}=n_{0} / 2 \omega_{0}$, where $\omega$ is the microwave field frequency and $\omega_{0}=\omega n_{0}^{3}$ is the rescaled frequency; therefore, in order to increase $N_{I}$ it is either necessary to increase the initially excited principal quantum number $n_{0}$, or to decrease the rescaled frequency $\omega_{0}$. On the other hand, in real experiments $n_{0} \lesssim 100$ and the additional condition $\omega_{0}>1$ must be verified if the system is to be found within the classically diffusive region (for sufficiently high field intensity $\epsilon$ ). In practice one typically obtains $N_{I} \approx 20$.

An interesting possibility for increasing $N_{I}$, thus allowing a better numerical and experimental analysis of dynamical localization, would be the consideration of a hydrogen atom within superimposed magnetic and microwave fields. Indeed, such a model, for a sufficiently strong magnetic field, displays classical chaotic dynamics even in the absence of the microwave field [8,9]. When the microwave field is turned on, this pre-existing chaos immediately leads to the onset of classical diffusive energy excitation, even when the microwave field intensity $\epsilon$ goes to zero. Such excitation takes place even when $\omega_{0}<1$, therefore the quantity $N_{I}=n_{0} / 2 \omega_{0}$ can be significantly increased.

Quantum effects may lead to localization of classical diffusion, hence the critical microwave field intensity required for ionization or the so-called quantum ionization border, will be determined by the condition $\ell_{\phi} \geq N_{I}$. Indeed, only when the localization length $\ell_{\phi}$, 
expressed in number of photons, is larger than the number $N_{I}$ of photons required for ionization, will a non negligible amount of quantum probability fall into the continuum states, thus leading to ionization.

In order to analyze the ionization process we consider the simple case in which both fields are parallel. Due to the cylindrical symmetry, the magnetic quantum number $m$ is an exact integral of the motion, and the Hamiltonian has the form:

$$
H=\frac{p_{z}^{2}}{2}+\frac{p_{\rho}^{2}}{2}+\frac{m^{2}}{2 \rho^{2}}+\frac{\omega_{L} m}{2}+\frac{\omega_{L}^{2} \rho^{2}}{8}-\frac{1}{\sqrt{z^{2}+\rho^{2}}}+\epsilon z \cos \omega t
$$

where $\omega_{L}=B / c=B($ Tesla $) / B_{0}$ is the rotation frequency in a magnetic field, $B_{0}=2.35 \times$ $10^{5}$ Tesla, $\epsilon$ and $\omega$ are the field intensity and frequency respectively (atomic units are used).

In the presence of a magnetic field only $(\epsilon=0)$, according to well-known results [8,9], the motion becomes strongly chaotic when $\omega_{L} n_{0}^{3} \gtrsim 4$ and no islands of stability are observed for $\omega_{L} n_{0}^{3} \approx 9$. When the microwave field is turned on, diffusive excitation of the electron will take place and the energy diffusion rate $D_{B}$ per unit time can be estimated in the following way: from eq. (11), $\dot{E}=-\epsilon z \omega \sin \omega t$ and therefore, for $\omega_{0} \ll 1$ in a quasilinear approximation [10], the diffusion is determined by the spectral density of perturbation. That is, $D_{B} \sim(\epsilon z \omega)^{2} / \delta \omega \sim(\epsilon z \omega)^{2} n_{0}^{3} \sim D_{0} \omega_{0}^{2}$, where $\delta \omega \simeq n_{0}^{-3}$ is a typical frequency at $\epsilon=0$ and $D_{0}=\epsilon^{2} n_{0} / 2$ is the diffusion rate in the chaotic regime for $B=0$ and $\omega_{0}=1$ [11. Therefore:

$$
D_{B}=\chi_{1} D_{0} \omega_{0}^{2}
$$

where $\chi_{1}$ is a constant to be numerically determined. This estimate is a good approximation if $\omega_{L} n_{0}^{3}$ is not too large (the case $\omega_{L} n_{0}^{3} \gg 10$ should be separately studied).

In the regime $\omega_{0} \gg 1$ the energy change will take place only when the electron passes close to the nucleus and, in analogy with the case $B=0$, the diffusion rate is given approximately by [11] $D_{B} \simeq \chi_{2} \epsilon^{2} n_{0} \omega_{0}^{-4 / 3} / 2=\chi_{2} D_{0} \omega_{0}^{-4 / / 3}$.

Notice that $D_{B}$ does not explicitly depend on the magnetic field $B$; however, the quantities $\chi_{1}, \chi_{2}$ can both weakly depend on $B$ for $\omega_{L} n_{0}^{3} \gtrsim 2$ (see fig 3 ). 
In order to check the above analytical estimates for $D_{B}$, we have carried out numerical simulations of the classical problem (10). We fixed the magnetic number $m=0$ and measured the energy excitation for an ensemble of 100 trajectories with the same initial energy $E_{0}=$ $-1 /\left(2 n_{0}^{2}\right)$. In fig. [ 1 we demonstrate the diffusive excitation process in energy for scaled frequency $\omega_{0} \ll 1$ and very small microwave field $\epsilon_{0}=\epsilon n_{0}^{4}$. Here $\omega_{L} n_{0}^{3}=9$ so that the motion is fully chaotic even for $\epsilon_{0}=0$.

The frequency dependence of the diffusion rate $D_{B}$ is shown in fig. 2 2 for fixed $\omega_{L} n_{0}^{3}=9$. The behavior for small and large frequencies is in agreement with the above theoretical estimates and is indicated by the two straight lines with $\chi_{1} \approx 18$ and $\chi_{2} \approx 2$ respectively. An interesting feature is that in a rather large frequency interval $\left(10^{-1} \lesssim \omega_{0} \lesssim 10\right)$ the frequency dependence is rather weak. We also checked the $\epsilon^{2}$ dependence of the diffusion rate $D_{B}$ by varying the field intensity over a few orders of magnitude.

In fig. 3 we plot the dependence of $D_{B}$ on magnetic field intensity for small values of $\omega_{0}$. According to our expectations, the diffusion rate remains approximately constant for $\omega_{L} n_{0}^{3}>2$ while below this value the internal motion becomes integrable and diffusion drops to zero.

The classical diffusive process will lead to ionization of the atom after a time $t_{I} \approx E_{0}^{2} / D_{B}$. For low field strength this time is much larger than both the orbital period and the microwave field period, and therefore quantum interference effects can lead to localization of classical diffusion. The localization length $\ell_{\phi}$ (in number of photons) can be obtained by using known estimates [12], according to which $\ell_{\phi} \sim \Gamma \rho$, where $\rho$ is the density of coupled states and $\Gamma$ is the one-photon transition rate, which in turn can be related to the classical diffusion rate via $\Gamma=D_{B} \omega^{-2}$. The striking advantage of such an approach to the computation of the localization length [12] is that it involves only classical characteristics of motion, namely the classical diffusion rate and density of coupled states. It is therefore sufficient to know only these two quantities to understand the properties of quantum dynamics. In this way one need not worry about quantum matrix elements, the structure of eigenfunctions and other quantum aspects. This fact becomes even more remarkable if one remembers that without a 
magnetic field the effective dynamics is well described by a one-dimensional atom model [11] while in a strong magnetic field the motion is essentially two-dimensional and quite different from the usual Kepler dynamics.

For zero magnetic field the effective density of coupled states is $\rho=n^{3}$, due to Coulomb degeneracy and the existence of an additional approximate integral of motion [11]. Instead, for sufficiently large $B$, the internal motion is chaotic and the density of coupled states is $\rho=n^{4}$. Therefore, in the latter case the localization length in number of photons is:

$$
\ell_{B}=\ell_{\phi} \frac{D_{B}}{D_{0}} \frac{n_{0}}{\omega_{0}^{2}}
$$

where $\ell_{\phi}=3.3 \epsilon_{0}^{2} n_{0}^{2}$ is the localization length in the microwave field only $(B=0)$ at $\omega_{0}=1$ and it is assumed that $l_{B}>1$.

For $\omega_{0} \ll 1$ we obtain from (3) $\ell_{B}=3.3 \chi_{1} \epsilon_{0}^{2} n_{0}^{3}$. The delocalization border, namely the critical microwave field intensity $\epsilon_{1}$ above which ionization takes place, is given by the condition $\ell_{B}=N_{I}$ leading to:

$$
\epsilon_{01}=\frac{1}{n_{0} \sqrt{6.6 \chi_{1} \omega_{0}}}
$$

which is well below the delocalization border within the microwave field alone. We would like to stress that the above estimates are valid in the regime $\omega \rho>1$ [12] that is $\omega_{0}>n_{0}^{-1}$ (and therefore the border (4) is valid for $n_{0}^{-1}<\omega_{0}<1$ ).

The above estimates were made in analogy to the problem of photonic localization in a random molecular quasi-continuum [12]. However, it is possible that such approach should be modified for the present case of a well defined quasiclassical system with adiabatically slow perturbation. Indeed, in such a situation, one can make a transformation to an instantaneous time basis in which transition matrix elements are proportional to the frequency of the perturbation. Here, many levels will be mixed only if this effective matrix element $V_{\text {eff }}$ proportional to $\omega$ is larger than the level spacing in one atomic shell $n^{-4}$. For a chaotic case we can estimate $V_{\text {eff }} \sim \epsilon \omega n^{5} / \sqrt{n}$ so that the diffusive exitation is quasiclassical (nonperturbative) only if $V_{e f f}>1 / n^{4}$. The same estimate can be also understood in the following 
quasiclassical way. The diffusion rate in the number of shells (the change of the principal quantum number in the unperturbed hydrogen $\Delta n=1)$ is $D_{n}=D_{B} n^{6}$. All the levels in the shell will be resolved after time $t_{s h} \approx 2 \pi n^{4}$. Therefore, the adiabatic perturbation is perturbative if the number of shells mixed during this time is less than $1:(\Delta n)^{2} \approx D_{n} t_{s h}<1$. The situation is quasiclassical in the opposite case $(\Delta n)^{2}>1$ when:

$$
\epsilon_{0}>\epsilon_{0 a} \approx\left(\frac{1}{3 \chi_{1}}\right)^{1 / 2} \frac{1}{\omega_{0} n_{0}^{3 / 2}}
$$

In this regime the diffusion rate measured in the number of shells per unit of time $\tau$ measured in units of $t_{s h}$ is $D_{s h}=(\Delta n)^{2} / \Delta \tau \approx 3 \chi_{1} \epsilon_{0}{ }^{2} \omega_{0}{ }^{2} n_{0}^{3}>1$. Similarly to the standard estimate 12 this diffusion will be localized after time $\tau^{*} \approx D_{s h}$ with localization length $l_{n} \approx \Delta n\left(\tau^{*}\right) \approx$ $D_{s h}$ (in the number of photons this gives $\left.l_{B}=l_{n} / \omega_{0}=D_{s h} / \omega_{0}\right)$. Then the delocalization border is determined by the condition $l_{n} \sim n_{0} / 2$ giving

$$
\epsilon_{0}>\epsilon_{0 q a} \approx \frac{1}{\sqrt{6 \chi_{1}} \omega_{0} n_{0}}
$$

As we see these adiabatic estimates are different from the above eqs. (3), (4). In our opinion the physical situation is more correctly described by the approach (3) - (4). However, only direct quantum simulations or laboratory experiments can lead to more definite conclusions.

For $\omega_{0} \gg 1$ the situation is more standard and we have:

$$
\ell_{B}=3.3 \chi_{2} \frac{\epsilon^{2}}{\omega^{10 / 3}} n_{0}
$$

and the condition $\ell_{B}=N_{I}$ leads to:

$$
\epsilon_{02}=\frac{\omega_{0}^{7 / 6}}{n_{0} \sqrt{6.6 \chi_{2}}}
$$

which is $\sqrt{n_{0} \chi_{2}}$ times smaller than the quantum delocalization border for $B=0$.

It is important to remark that, contrary to the case $B=0$, the localization length depends now on photon number. Indeed, for $\omega_{0} \ll 1, \ell_{B} \propto n_{0}^{11} \sim\left(N_{I}-N\right)^{-11 / 2}$ while for $\omega_{0}>1, \ell_{B} \propto n_{0} \sim\left(N_{I}-N\right)^{-1 / 2}$. This means that localization length becomes larger near the ionization border. However, this effect is not important if $\ell_{B} \ll N_{I}$ while for $\ell_{B} \approx N_{I}$ it will lead to a change in the numerical factor for the delocalization border. 
We also remark that for $\omega_{0} \ll 1$ the quantum delocalization border $\epsilon_{01}$ will be relevant only if it remains smaller than the ionization border for a static electric field in the presence of a parallel magnetic field.

Finally, we would like to stress that the problem under investigation represents a qualitatively new situation as compared to the hydrogen atom in a microwave field only. Therefore, the above preliminary estimates require a detailed check by quantum simulations.

On qualitative grounds, the effects described in the present paper can also take place in non hydrogen atoms in a static electric field and in the absence of a magnetic field. It is indeed known that for non hydrogen atoms in static electric fields, the eigenfunctions can be approximately ergodic and the level spacing statistics shows level repulsion of the Wigner-Dyson type [13]. This situation is similar to that in which internal chaos is present due to a strong magnetic field. Since in the formulae derived in this paper the magnetic field dependence is quite weak, we expect that they are approximately valid even for non hydrogen atoms in static electric fields. However, one should keep in mind that while qualitatively the ionization process should behave similarly to the case with a magnetic field the chaos properties in a static electric field are not so strong and therefore this case should be analyzed in more detail. Such a situation with atoms in a static electric field is quite interesting since it is more suitable for laboratory experiments. Finally, we believe that the results described in the present paper will not be significantly changed if the electric and magnetic fields are not parallel, provided that the electric field is relatively weak. The reason being that the magnetic quantum number $m$ will determine the existence of an approximate integral of motion similar to the case of hydrogen in a microwave field [11].

In this paper we have introduced and discussed a new model that should allow for a clear experimental investigation of quantum dynamical localization. Its main feature is the wide range inside which it is possible to change physical parameters such as $\omega_{0}, \epsilon_{0}$ and the interaction time. A possible convenient choice of parameters is $n_{0}=60, \omega_{0}=0.1$ ( $\omega=3 . \mathrm{GHz}), B=10 T\left(\omega_{L} n_{0}^{3}=9\right)$ [or static electric field $\epsilon_{0 s t} \approx 0.05$ for non hydrogen atoms]. For these conditions we have $\epsilon_{01}=0.005, N_{I}=300$ and interaction time (measured 
in number of microwave periods): $\tau_{I}=\omega t_{I} / 2 \pi=1 /\left(4 \pi \chi_{1}\right) \cdot 1 /\left(\epsilon_{0}^{2} \omega_{0}\right) \approx 1800$. If, however, we define the ionization probability as the occupation probability of levels $n>70$ then the number of photons will be $N_{I} \sim 100$ and the ionization time $\tau_{I}=180$ microwave periods. This range of parameters seems to be suitable for laboratory experiments. 


\section{FIGURES}

FIG. 1. Example of diffusive energy excitation $\left\langle\Delta E^{2}\right\rangle / E_{0}^{2}$ with time, for parameter values $\omega_{L} n_{0}^{3}=9, \omega_{0}=0.05$ and $\epsilon_{0}=0.003$. The straight line shows the diffusive growth with a fitted rate given by eq.(2) with $\chi_{1}=9.8$.

FIG. 2. Dependence of scaled diffusion rate $D_{B} / D_{0}$ on scaled frequency $\omega_{0}$ for $\omega_{L} n_{0}^{3}=9$. Here $D_{0}=\epsilon_{0}^{2} / 2 n_{0}^{7}$ and $\epsilon_{0}$ in the interval $10^{-3} \leq \epsilon_{0} \leq 10^{-2}$. The straight lines show the theoretical dependence for $\omega_{0} \gg 1$ and $\omega_{0} \gg 1$ with $\chi_{1}=18$ and $\chi_{2}=2$ respectively (see text). Ensembles from 30 to 100 orbits have been used.

FIG. 3. Scaled diffusion rate $D_{B} / D_{0}$ as a function of the scaled frequency $\omega_{L} n_{0}^{3}$ for $\omega_{0}=0.05$ and $\epsilon_{0}=0.003$. 


\section{REFERENCES}

(a) also at Istituto Nazionale di Fisica della Materia, Sezione di Milano

(b) also Budker Institute of Nuclear Physics, 630090 Novosibirsk, Russia

[1] Quantum Chaos: a selection of papers compiled and introduced by Giulio Casati and Boris Chirikov. (Cambridge University Press, 1995).

[2] G. Casati, B.V. Chirikov, J. Ford and F.M. Izrailev, Lecture Notes in Physics 93 (1979) 334. This paper is also included within [1].

[3] E.J. Galvez, B.E. Sauer, L. Moorman, P.M. Koch and D. Richards, Phys. Rev. Lett. 61 (1988) 2011.

[4] J.E. Bayfield, G. Casati, I. Guarneri and D.W. Sokol, Phys. Rev. Lett. 63 (1989) 364.

[5] M. Arndt, A. Buchleitner, R.N. Mantegna, H. Walther, Phys. Rev. Lett. 67 (1991) 2435.

[6] F.L. Moore, J.C. Robinson, C. Bharucha, P.E. Williams and M.G. Raizen, Phys. Rev. Lett. 73 (1994) 2974.

[7] G.P.Collins, Physics Today 48, N 6 (1995) 18.

[8] D. Delande in Chaos and Quantum Physics edited by M.--J. Giannoni, A. Voros and J. Zinn-Justin (North-Holland, Amsterdam, 1991), p. 665.

[9] H. Friedrich and D. Wingten, Phys. Rep. 183 (1989) 37.

[10] A.J. Lichtenberg and M.A. Lieberman, Regular and Chaotic Dynamics (Springer, Berlin 1992).

[11] G. Casati, I. Guarneri and D.L. Shepelyansky, IEEE Journal of Quantum Electronics, 24 (1988) 1420.

[12] D.L. Shepelyansky Physica D 28 (1987) 103. 
[13] D. Delande, talk given at the Adriatico Research Conf. on Chaos in atomic and molecular systems Trieste, 1995. 
Fig. 1

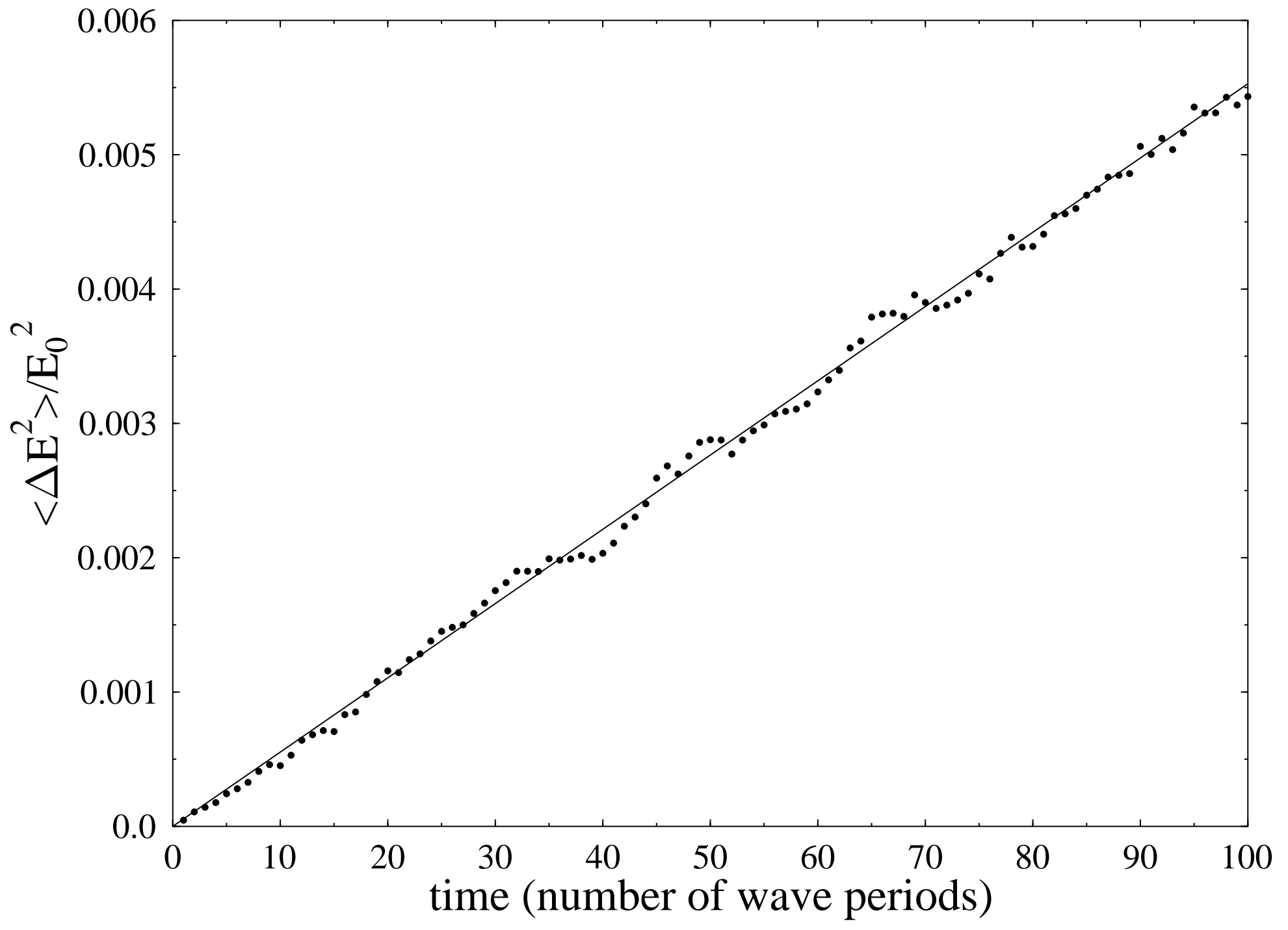


Fig. 2

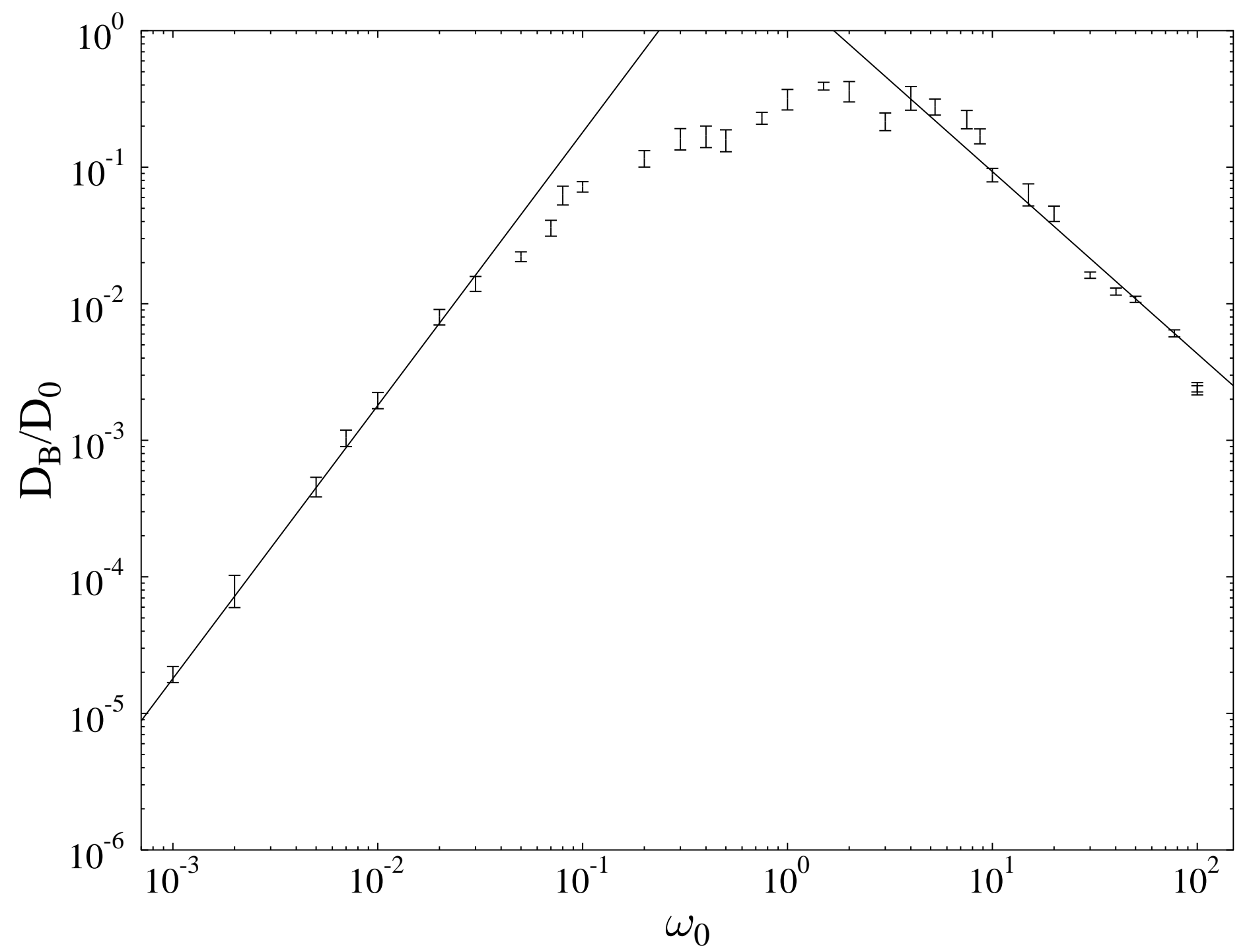


Fig. 3

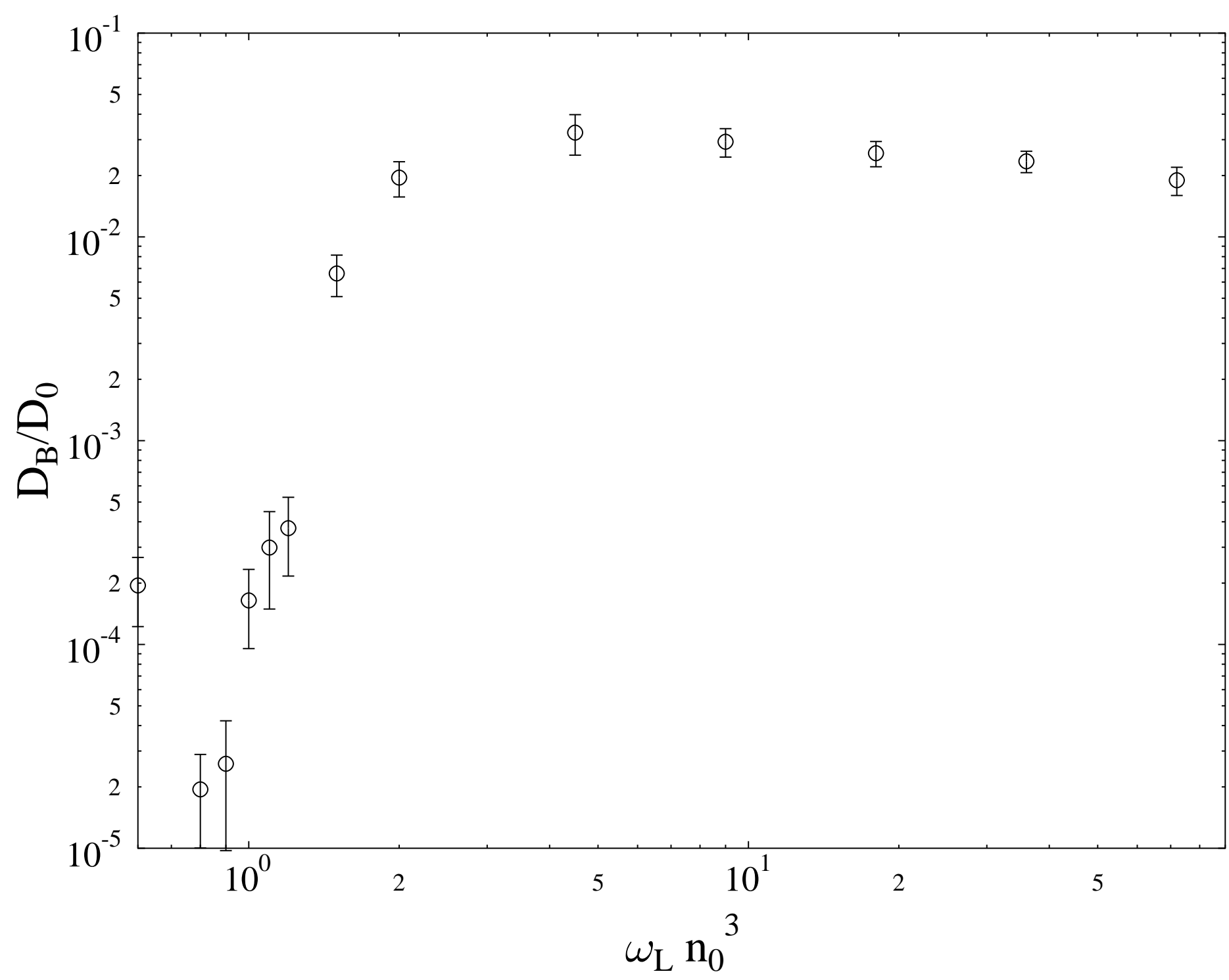

